\title{
Social networks and academic performance in physics: Undergraduate cooperation enhances ill-structured problem elaboration and inhibits well-structured problem solving
}

\author{
Javier Pulgar $\odot^{*}$ \\ Departamento de Física, Universidad del Bío Bío, Concepción 4051381, Chile \\ Cristian Candia ${ }^{\dagger}$ \\ Kellogg School of Management, Northwestern University, Evanston, Illinois 60208, USA; \\ Northwestern Institute on Complex Systems (NICO), Northwestern University, \\ Evanston, Illinois 60208, USA \\ and Centro de Investigación en Complejidad Social (CICS), Facultad de Gobierno, \\ Universidad del Desarrollo, Las Condes 7550000, Chile \\ Paul M. Leonardi \\ Technology and Management, College of Engineering, University of California, \\ Santa Barbara, California 93106-5129, USA
}

(Received 23 December 2019; accepted 18 May 2020; published 11 June 2020)

\begin{abstract}
For several decades, scholars have studied the role of cooperation and its outcomes in educational contexts. Yet we lack a complete understanding of how different instructional strategies impact the relationship between cooperation and learning. In this paper we present results from a field experiment with 82 first-year students in an introductory physics course showing how different instructional strategies led to different social configurations in the classroom and to differences in individual academic performance. Surprisingly, we found that students who actively sought out information from multiple peers were less likely to perform well on well-structured problems as compared to those who did not seek help, whereas, for ill-structured (real-world-like) problems, this effect depended on the features of the learning environment. We observed that good performance on ill- and well-structured problems was sensitive to different social network configurations. In a highly clustered network (which contains redundant information), students performed better on well-structured problems than ill-structured problems. By contrast, students with access to network structural holes (which enable access to more diverse information) performed ill-structured problems better than well-structured problems. Finally, ill-structured problems promoted creative thinking, provided that instructors guided the problem-solving process and motivated students to engage in the appropriate cognitive demands these problems entailed. Our results suggest that teaching and instructional strategies play an important role in cooperative learning; therefore, educators implementing cooperative learning methods have to accompany them with adequate instructional strategy.
\end{abstract}

DOI: 10.1103/PhysRevPhysEducRes.16.010137

\section{INTRODUCTION}

Cooperation is fruitful for learning [1,2], leading to better academic outcomes in educational contexts [3-7]. Moreover, cooperation among peers eases collective learning leading to the emergence of good ideas, while promoting processes linked with crucial competencies in today's

\footnotetext{
*jpulgar@ubiobio.cl

cristian.candia@kellogg.northwestern.edu
}

Published by the American Physical Society under the terms of the Creative Commons Attribution 4.0 International license. Further distribution of this work must maintain attribution to the author(s) and the published article's title, journal citation, and DOI. society [8]. Yet, little is known about how different instructional strategies impact the relationship between cooperation and learning.

In this study we investigated students' social networks from three different classes of an introductory physics course and determined the social structures that facilitate good performance on well-structured physics problems and those that facilitate performance on ill-structured physics problems [9]. By well-structured problems, we refer to learning tasks that are solved through algebra, often characterized in physics education research (PER) by simplified and idealized situations that have little to no connection with students' real-world experience [10], and frequently found in physics textbooks [11,12]. By illstructured problems, we refer to those problems associated 
with real-world problems [13] that lack the information that individuals would use to find an already known and unique solution. Ill-structured problems introduce high levels of uncertainty associated with a spectrum of possible strategies on how to proceed to develop a solution $[9,14]$. In our study, the ill-structured task consisted of student groups generating physics problems for high school students.

Through this study, we explored whether different collaborative mechanisms - creative combinations (CC) [15-18] or interrogation logics (IL) [19]—predict good performance on well- and ill-structured problems. To this aim, we used three different experimental conditions for 82 first-year students, where we varied teaching and instructional strategies. The experiment was run in an introductory physics course over two months at a university in Northern Chile. To explore the extent to which students' social structures facilitated academic performance, we collected data on students' performance on a physics test designed with well-structured problems, and performance on an ill-structured problem. In addition, we asked students to respond to an online peer-nomination survey related to their social interactions for seeking information to solve problems. Finally, we tested the effects of different instructional strategies on academic performance and whether student collaboration was sensitive to the learning environment.

\section{A. Physics problem solving and collaboration}

Well- and ill-structured problems have different characteristics that might necessitate different forms of collaboration. In physics education, well-structured problems (i.e., well-structured tasks) demand the use of a limited number of rules and principles (e.g., algebra and physics principles), along with a set of procedures that are well organized and constrained to certain parameters (e.g., initial and/or the final conditions on a motion problem in kinematics). These tasks have predictable actions that are frequently used to solve similar problems [20,21]. Good performance on well-structured problems has not been reported necessarily as a consequence of conceptual understanding $[22,23]$, as students tend to solve such tasks through a "plug and chug" strategy [12,20,23]. In addition, wellstructured problems can be defined as disjunctive tasks [24] with low levels of positive interdependence [1], as these tasks might be solved by the most capable or vocal students when addressed in groups.

On the other hand, the difficulty in solving ill-structured problems lies in deciding the appropriate constraining conditions that would guide solvers to transition from the open-ended scenario towards strategies to come up with their unique response [25]. Fortus [13] studied the importance of making assumptions when solving illstructured mechanics problems among experts and novices. $\mathrm{He}$ found that even experts struggled with deciding adequate assumptions about the physics variables and principles involved, and about the absolute or relative magnitudes of the variables for deciding on and developing solutions. From the embedded attributes of ill-structured problems, one might expect ill-structured problems to introduce high levels of positive interdependence [1], and be perceived as additive tasks [24], where performance emerged as the sum of all members' contributions and relevant abilities [26]. These expectations are coherent with the experience from Heller and colleagues [27], who designed context-rich problems as an alternative to traditional textbook physics activities [10], and found that groups performed better than isolated students.

\section{B. Network centrality and learning}

Social network theory provides two alternative collaborative mechanisms that enable knowledge development and idea generation for problem solving: (i) creative combinations (CC) and (ii) an interrogation logic (IL), where both collaborative mechanisms are oriented towards the emergence of good ideas but through different social configurations. The former, $\mathrm{CC}$ of information is a mechanism that depends on one's structural position within the network of students, and the diversity of knowledge that could be accessed through such structure. Accordingly, good ideas would depend on how people learn new information through different social ties, from zones of high knowledge redundancy (i.e., high network cohesion), to zones where actors have access to isolated partitions of the network (i.e., structural holes). Actors who bridge connections between two unconnected individuals or groups, or who span structural holes through brokerage, would enjoy the advantages of social capital by accessing the resources available in different places of the network and are therefore more likely to produce creative ideas [15,28]. In addition, individuals and groups located in central positions of the social network are more likely to have creative outputs, because they are placed in paths connecting two or more teams, and therefore have access to the information that is transferred through those links [15,28-30]. In contrast, peripheral students and groups placed at the end of the information path would depend on central groups letting the knowledge flow in the peripheral group's direction, thus making them less likely to be able to take faster advantage of the information flowing throughout the network [31].

Differently, the IL [19] is a mechanism where highly constrained networks (e.g., cohesive groups) afford opportunities for creative ideas, but through different cognitive processes than actors who span structural holes. On tasks that benefit from IL, subjects' attention is focused on specific content and the contents' related ideas rather than on the diversity of information flowing throughout the network. This process enables an in-depth examination of the local knowledge among the individuals embedded in the cohesive group.

In education and PER literature, we found no reference to whether the aforementioned collaborative mechanisms 


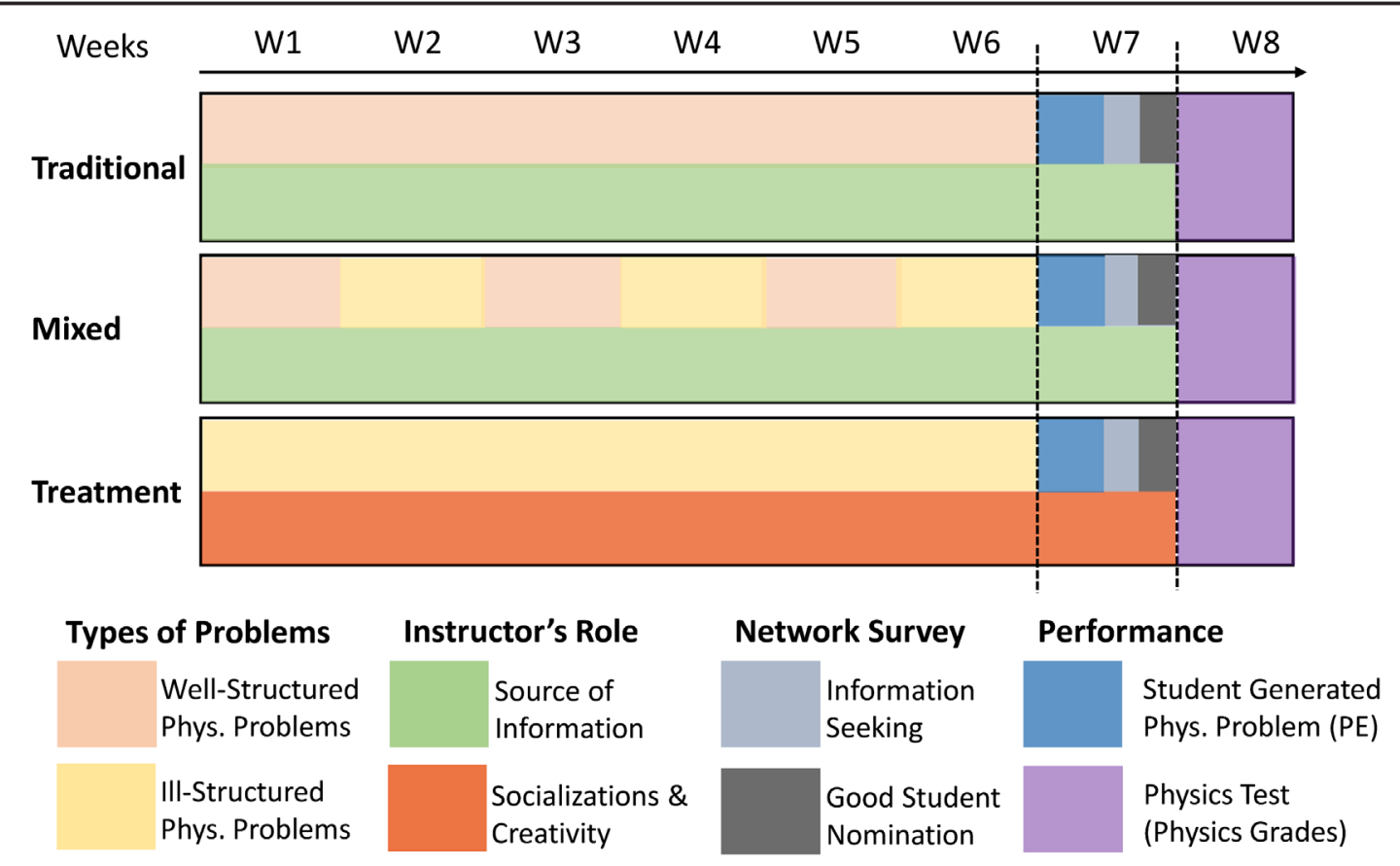

FIG. 1. Experiment design, with three sections: traditional, mixed and treatment. The diagram shows the timeline of the events from weeks 1 through 8 . Includes the unique instructional characteristics per section (types of problems, and instructor's role), along with performance instruments and network surveys.

(i.e., CC and IL) are advantageous for solving different types of problems. So far, education researchers have used network analysis to explore the academic advantages of central positions in students' networks. Academic performance is most likely enhanced by being immersed in a cohesive social network from which students can take advantage of the information, skills, and abilities of others that might be shared through social ties [28,32-36]. Research evidence has found significant correlations between centrality measures and performance [37,38]. Moreover, teaching and learning conditions play an essential role in encouraging (hindering) student social interaction or for students to reach central positions in a classroom network $[39,40]$. Finally, recent evidence has found that the number of social ties (e.g., centrality) is not a straightforward predictor for academic achievement. More outgoing social ties (i.e., from ego to alter) for cooperation showed a negative effects on students' performance, while reciprocal ties led to better academic performance [7], thus adding important conditions over the nature of the social relationship for academic achievements.

From this body of evidence, we asked the following: What role do instructional strategies (teaching and learning activities) play in cooperation and learning outcomes in an undergraduate physics course?

\section{METHODS}

We conducted a field experiment in three undergraduate sections of an introductory physics course designed for engineering majors at a university in Northern Chile. The experiment lasted during an 8 week period in 2018. We aimed to explore whether student collaboration had similar or different effects on performance on well- and illstructured physics problems. For this purpose, in collaboration with course instructors, we designed a battery of ill-structured problems grounded in real-life situations for a weekly administration during problem-solving sessions for a period of 7 weeks.

Students were first- or second-year engineering majors, pursuing careers in either Industrial Civil Engineering or Software Civil Engineering. A total of 82 students participated in the study.

The details of the experiment are depicted in Fig. 1. Here, we show the instructional characteristics of the three sections (traditional, mixed and treatment), the types of physics problems they worked on, and the role each instructor enacted in guiding the sessions. Of the 82 students in the study, 33 students were in the traditional class, 23 in the mixed class, and 26 in the treatment class. In terms of the instructor's role, we assigned two alternative behaviors: (a) source of information, that is, the instructor facilitated direct information to respond to students' questions regarding the problem; and (b) socialization and creativity, that is, the instructor responded to students questions by directing their attention to other classmates who may have either asked a similar question, or responded to the question already. Figure 1 also shows performance and network instruments administered in weeks 7 and 8. More details of these instruments are in the following section. 


\section{A. Data collection}

During the 7th week of the experiment (Fig. 1), we tasked students with the activity of designing a physics problem for high school students (see the activity instructions in Supplemental Material [41]) addressing the concepts and principles of circular motion. At the end of the session we gathered students' generated physics problems, and asked them to respond to an online peer-nomination survey. We used this survey to identify the social network of the class during the problem solving session where participants solved the ill-structured problem.

\section{Network surveys}

The survey consisted of two questions administered through Qualtrics online survey service that aimed to measured the following networks (see survey design in Supplemental Material [41]):

a. Network of information seeking: From whom have you sought information to solve the physics problem addressed in this session?

b. Network of good students: Who is a good physics problem solver in your class? (i.e., a student you believe is good at understanding physics content and solving physics problems).

To facilitate students' responses on each of these questions, we included the roster of students enrolled per section. Consequently, subjects responded by selecting the individuals in their sections from whom they sought information, as well as the ones that were perceived as good students. Both questions led to the construction of directed (i.e., ties are not necessarily reciprocal) and binary networks [i.e., links between nodes either exist (1) or do not exist (0)]. The network of information seeking was designed to reveal whether students engaged in social interactions for the purpose of finding resources and ideas for solving the ill-structured physics problem. Because information flowing through social ties (i.e., flow ties) is difficult to measure, social interactions such as "seeking information" may be perceived as proxies of information flow [30,42]. Generate a "good student" network is thought to enable an additional dimension, revealing what type of students engaged in information seeking, to then explore whether this perceived prestige is a valuable contributor to the social processes that affect academic success.

\section{Dependent variables}

Instructors of the course determined physics grades, the first dependent variable, which were students' scores on a test designed by instructors which consisted of three well-structured problems administered in week 8 (Fig. 1). These grades were used in the analysis as measurement of performance on well-structured physics problems. Physics grades were shared by the instructors three weeks after the day of data collection, without the possibility for us to review the assessment instrument or the students' specific solutions to these problems.

Finally, the performance measure for students' solutions to ill-structured problems is labeled as problem elaboration (PE). PE was constructed to assess the degree of elaboration in students' generated problems for the content of the kinematic of circular motion. The following is an example of a problem generated by one student group:

Donkey Kong wants to throw barrels to King K Rool. For this, Donkey Kong throws one barrel with an angular speed of $2 \pi \mathrm{rad} / \mathrm{s}$. By knowing that at $3 \mathrm{~s}$ its speed is $10 \pi \mathrm{rad} / \mathrm{s}$, and that the barrel impacts at $5 \mathrm{~s}$, determine: (a) the angle covered by the barrel; (b) the magnitude of the centripetal and tangential acceleration at the moment of impact at $6 \mathrm{~cm}$ from its center; and (c) frequency and period.

Because creative tasks and their respective outputs may deviate from standard solutions, analyzing performance on ill-structured problems was conducted through the identification of embedded features and characteristics within the problem. We conducted the analysis on a total of 26 problems (traditional $=10$; mixed $=9$; treatment $=7$ ). In order to conduct the analysis, we translated these problems from Spanish to English, which were revised by a native English speaker knowledgeable in physics. The analysis of these solutions (i.e., physics problems) was conducted on NVivo 12 plus, a software for qualitative data analysis. This qualitative description came from the identification of problems' attributes and characteristics, such as physics concepts used as data and/or questions, type of information, contextual details, and word count as well as other variables shown in Table I. A first wave of problem coding was conducted by the lead author, which yielded an initial version of the code book, which was revisited in collaboration with a trained graduate student in qualitative analysis and physics content. (For more details, see Ref. [43].) After reaching agreement about the code book, an independent wave of coding was performed, where both the lead author and the trained graduate student covered $40 \%$ of the data (10 problems), obtaining a Cohen's kappa of 0.92 .

\section{Network measures}

The network measures used for this analysis were computed from the network of information seeking [i.e., constructed from response to survey question (a)]. This set of social structure variables consisted of different metrics of network centrality (degree, in-degree, out-degree, betweenness, and eigenvector), as well as network constraint and a brokerage metric known as gatekeeper. In what follows, we describe each of these variables:

- Degree is a network measure of centrality that counts the total number of edges (i.e., social ties) connecting the focal actor. 
TABLE I. Code description of problem characteristics for problem elaboration.

\begin{tabular}{|c|c|}
\hline Code & Description \\
\hline Physics concepts asked & Physics concepts used as problem items (e.g., angular speed, tangential acceleration). \\
\hline \multicolumn{2}{|r|}{ 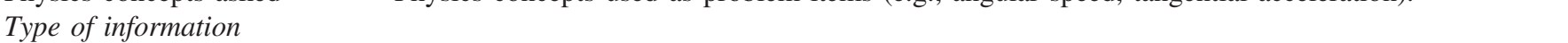 } \\
\hline Ready-to-use info & Data is explicitly presented in the problem and with appropriate units for its use. \\
\hline Conversion of units & Physical quantities that need conversion to respect the IS of units (i.e., $\mathrm{m}$ and s). \\
\hline Text to math & $\begin{array}{l}\text { Physics information is presented in written form and needs translation into mathematical } \\
\text { expressions (e.g., "begin its motion from rest" or "uniform motion"). }\end{array}$ \\
\hline Algebra transformation & Physics information for solving the problem needs algebraic steps for accessing and using it. \\
\hline Information research & The problem requires researching appropriate magnitudes to solve the problem. \\
\hline Assumptions & $\begin{array}{l}\text { Problem forces students to assume particular characteristics of the problem, } \\
\text { such as constant acceleration, or the position of the 'particle' that describes the circular motion. }\end{array}$ \\
\hline No. phys. concepts asked & Number of physics concepts used as problem items. \\
\hline No. equations needed & Number of equations required to solve the problem. \\
\hline Contextual details & Elements from real-life activities, and/or actors witnessing or engaging in actions. \\
\hline Word count & Number of words used on the problems' description. \\
\hline Cognitive demand & Taken from a taxonomy of introductory physics problems [44]. \\
\hline
\end{tabular}

- Out-degree: For directed networks this measure of centrality counts the number of outgoing edges or social ties for a given node, that is, the number of links directed from the focal actor towards other individuals within the network.

- Gatekeeper: A brokerage measure that counts the number of times node $i$ bridged connections between $j$ and $q$, where source node $j$ is a member of a different group than $i$ and $q$, which in turn are members of the same group. A gatekeeper broker is an individual that spans nonredundant ties with nodes outside its own group, has connections with its own group members, and engages in bringing information from the outside ties, while the destination of that information is a members within its own group. In Fig. 2, nodes $C, D$, and $F$ display such type of brokerage as they display ties with nodes outside their own units (sources), but at the same time engaged with teammates, and therefore, may have access to novel information from these outside sources and bring it to the group.

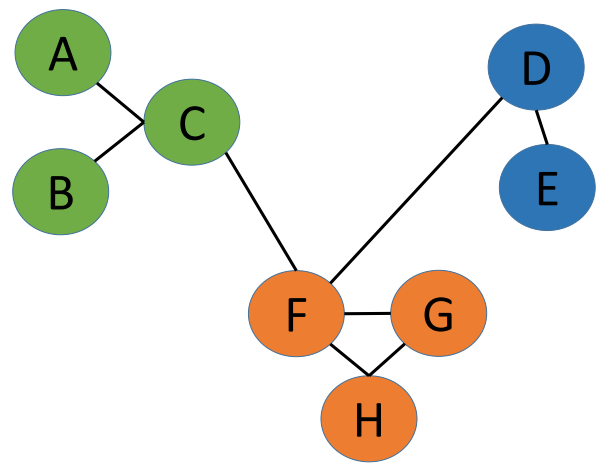

FIG. 2. Network diagram of constraints and structural holes. Node $F$ has access to different sources of information from blue and green communities.
- Eigenvector: network centrality measure that relates to social influence within a system, as it depends on whether the nodes tied to the focal actor show evidence of social ties to other well-connected nodes.

Accounting for the connectivity of one's friends is key for flow processes [42] to the extent that friends with social relationships outside one's social domain might boost chances of receiving and sharing valuable information for learning, innovation, and social status. The algebraic representation of eigenvector is as follows: $e_{i}=\lambda \sum_{j} x_{i j} e_{j}$. Here, $e_{i}$ is the eigenvector centrality of node $i$, and $\lambda$ is the largest eigenvalue of $e_{i}$. Moreover, $x_{i j}$ can take values of 1 or 0 depending on whether nodes $i$ connected to $j$ or not, respectively. That is, eigenvector centrality of node $i$ is proportional to the sum of its neighbors' eigenvector centralities.

- Constraint: Constraint is a network measure that accounts for the number of redundant social ties, that is, the degree to which a node spans ties with others who are also connected to each other [45].

This is an inverse measure of brokerage (the node that bridges isolated portions of the network, thus accessing structural holes). High constraint will indicate that a node is totally invested in a group of already connected others, and will therefore have access to zero structural holes. The definition introduced by Ref. [45] for constraint: $C_{i}=\sum_{j} c_{i j}, i \neq j$; $c_{i j}=\left(p_{i j}+\sum_{q} p_{i q} p_{j q}\right)^{2}, q \neq i, j$, where $C_{i}$ is the constrain of node $i$, and $c_{i j}$ an index that indicates $i$ 's investment on its relationship with $j$, counting direct ( $p_{i j}$ : proportion of tie strength between $i$ and $j$, relative to all of $i$ 's ties) and indirect $\left(\sum_{q} p_{i q} p_{j q}\right)$ : proportion of tie strength through indirect paths connecting $i$ and $j$ via $q$.

Network constraint is negatively associated with brokerage - the investment in social interactions that 
bridge connections between previously isolated portions of the network, known as structural holes. For instance, in Fig. 2, node $F$ has access to a structural hole because it shows nonredundant ties between groups green and blue, and may access new information and ideas from both groups, which may provide unique opportunities for creative combinations. Consequently, node $F$ would have lower network constraint than nodes $G$ and $H$ as these have redundant ties, and therefore are incapable of brokering beyond their close network.

\section{Control variables}

Finally, we accessed data on students' scores on a nationwide standardized test [University Selection Test or (UST)] to access educational information, type of high school from which students graduated, city where they lived before entering university, and engineering major and gender to utilize as control variables in our analysis. These control variables aim to account for the homophily mechanisms that drive social network configuration in higher education $[3,6,46]$.

\section{B. Data analysis}

After removing missing cases, the number of students remaining for analysis was $N=67$. We used ordinary least-squares multiple regressions (OLS) on the continuous dependent variables (i.e., physics grades and problem elaboration) to explore the effect of network structures, as well as differences in performance by sections.

First we tested the effect of network measures over problem elaboration and physics grades by regressing physics grades on network predictors. The models for grades and problem elaboration included interaction terms between class sections and the particular investigated network measure, which enable a comparison and interpretation of whether the network variable has a similar effect on the whole sample or was dependent on the learning environment defined by the type of problems and teaching strategy. In order to ease interpretation of regression coefficients, all predictors were standardized. For interpreting the regression coefficients of categorical variables such as academic sections (as), school type (st) and engineer major (em), readers must consider that the coefficient emerges as the difference between the variable in the model and the baseline categories (here as: traditional, st: industrial civil engineer, and em: public schools).

Later, we explored whether engaging in problem elaboration enabled good performance through the moderation of social engagement on information seeking. In other words, we investigated the degree to which ill-structured problems foster students' ability to answer well-structured problems in interaction with students' network structure. For this purpose, we fit OLS multiple regression models with an interaction term between problem elaboration and network measures.

Finally, to deal with the small sample size and test the robustness of our results, we used bootstrap random sampling [38] at 0.1 level, where we observed no important changes in our regression coefficients.

\section{RESULTS}

\section{A. The effect of social structures on physics grades}

Figure 3 depicts classroom networks for information seeking. The node size represents out-degree centralitythe number of times that the student seeks for information to a peer - whereas color shades indicate the grade obtained in the physics test (well-structured problem). By visually

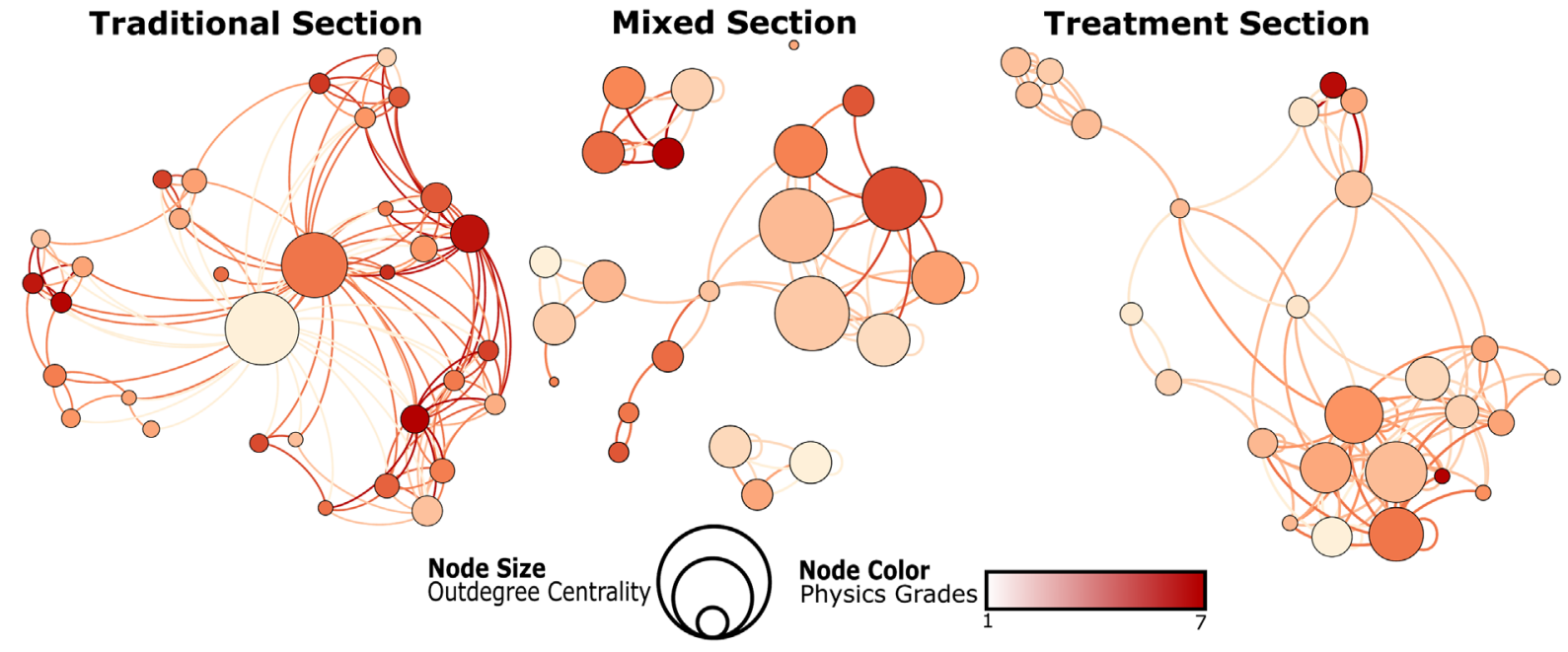

FIG. 3. Classroom networks for the three analyzed sections: traditional, mixed, and treatment. Node color represents physics grades (dark red being the highest), and the node size represents the out-degree centrality, i.e., the number of times that a student seeks information in the classroom. 
inspecting the figure, we observe students who received high grades - nodes with darker colors - tend to be smaller (i.e., lower out-degree) and located at the periphery of the network. In contrast, low grades students tend to have higher out-degree.

Figure 4 summarizes the multiple regression models fitted using $\log$ (out-degree) (a), gatekeeper (b), network constraint (c), $\log ($ degree ) (d), eigenvector (e), and the baseline of good student centrality (f). These models allowed us to explore the effect of network structures on physics grades, and whether such effects are invariant to the teaching conditions enacted on each section. For all models, we controlled for different confounding variables such as good student nomination; higher education application score (UST); a dummy variable that takes value 1 if students reside in the same city as their family; a dummy variable for gender; and a dummy variable for private or nonprivate high school.

The regression coefficient for the treatment section is positive and significant, with a large effect on physics grades in models (a)-(e), even after controlling for all the confounding variables. Physics grades for students under the treatment condition increased almost a point more than students in the traditional section would score under similar conditions. This result suggests important effects of the learning environment generated in the treatment section, based on ill-structured problems, along with guidance over socialization of information.

Surprisingly, and contrary to evidence found in the literature, centrality metrics showed a negative effect on grades. These effects are observed for $\log$ (out-degree) (a), gatekeeper (b), $\log ($ degree) (d), and eigenvector (e). Because out-degree refers to the number of outgoing ties, the activity of seeking out information was not found to be related to receiving good grades. In general, having a high number of social ties, either incoming or outgoing, was negatively related to physics grades, as seen in model 4(d) for $\log$ (degree). Consistent with our previous results, connecting others outside one's group for information seeking [Fig. 4(b)] does not afford academic success in well-structured problems.

Deviating from other models, the regression coefficient for network constraint in Fig. 4(c) is positive, yet not statistically significant. The direction of the coefficient is (a)

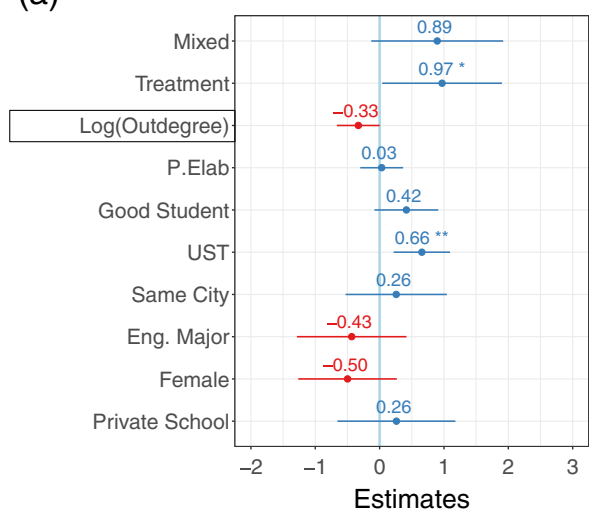

(d)

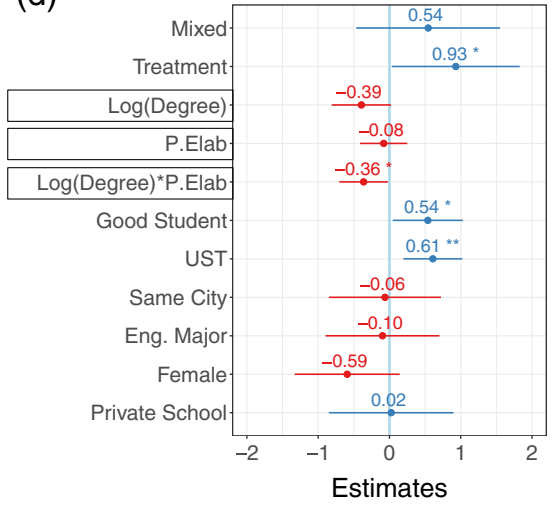

(b)

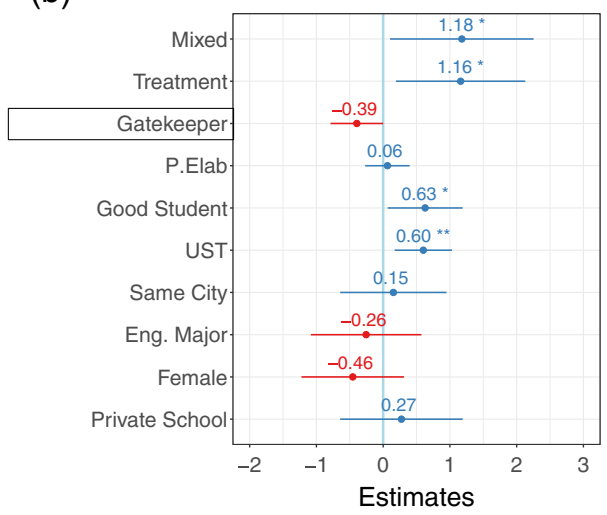

(e)

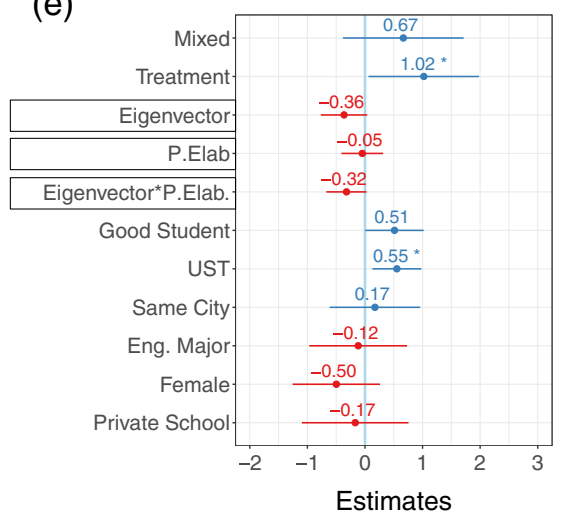

(c)

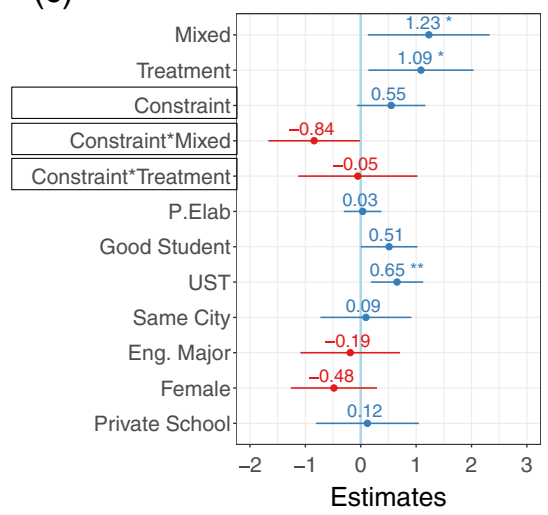

(f)

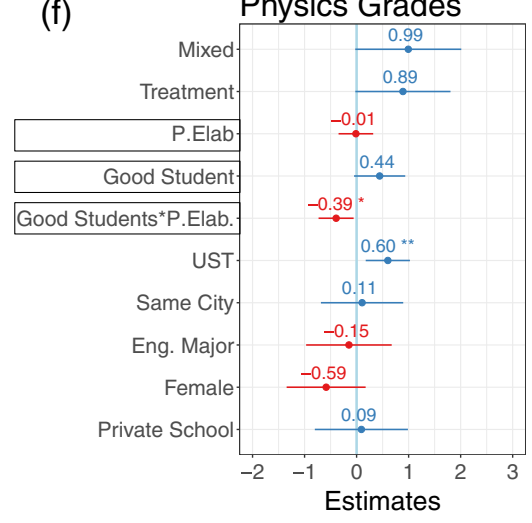

FIG. 4. Graphic depiction of OLS multiple regression models for physics grades regressed on network predictors, controlling for confounding variables (see Table 2 on the Supplemental Material [41] results section). Red indicates a negative effect and blue indicates a positive effect. One star $(*)$ indicates significance at a level of $p$ value $<0.05$ and two stars $(* *)$ indicate significance at a level of $p$ value $<0.01$. 


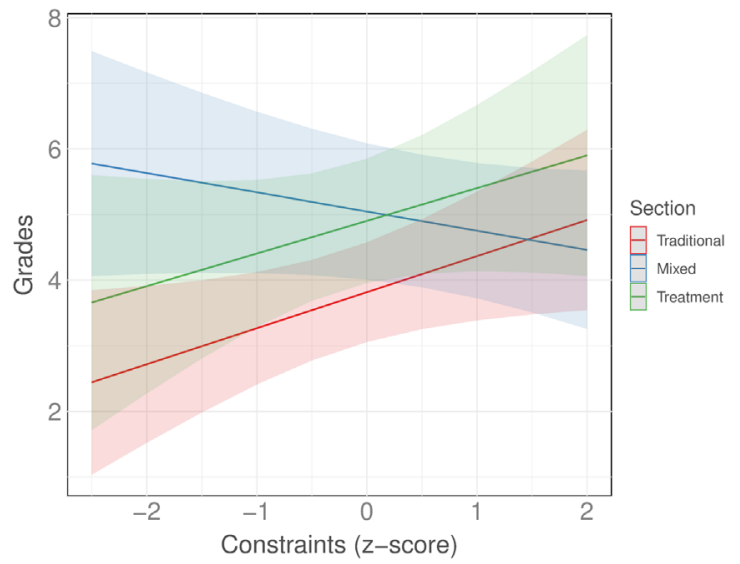

FIG. 5. Interaction between network constraint and sections for predicting physics grades.

consistent with the collaborative process of interrogation logic [19], a process that benefits from highly constrained networks. To disentangle this relationship, Fig. 5 shows the interaction between network constraint and classroom sections in predicting physics grades. According to the plot, both traditional (red) and treatment sections (green) show positive slopes that are not statistically (significantly) different, whereas the effect of network constraint is negative for the mixed section (blue). This result suggests that high access to structural holes (i.e., low constraint) leads to higher grades just for the mixed section.

\section{B. The effect of social structures over problem elaboration}

Figure 6 summarizes the multiple regression models on problem elaboration (See Supplemental Material [41],
Table 1), with the main predictors of academic section, network constraint [Fig. 6(a)], gatekeeper brokerage [Fig. 6(b)], and eigenvector centrality [Fig. 6(c)]. We also included the interaction between the network metric and the academic section variable to explore whether there were differences in problem elaboration due to differences in instruction.

Figure 6(a) reveals that network constraint value-being a member of a cohesive network with redundant ties-has a positive but nonsignificant effect on problem elaboration. Figure 6(b) reveals a negative and statistically significant difference in problem elaboration between the mixed and traditional section, while such difference is also negative but not significant between treatment and traditional. Moreover, students seeking out information from peers from other groups and sharing it with their team members (i.e., gatekeeper brokerage) is a positive predictor of problem elaboration above and beyond instructional differences. The interaction term is negative for the mixed compared to the traditional section and statistically significant at 0.001 level and less negative for treatment relative to traditional sections but at 0.1 level of significance. To understand this result, Fig. 7 depicts the relationship between problem elaboration and gatekeeper by section. Accordingly, the mixed section exhibits a negative slope, while being a gatekeeper in traditional and treatment sections yields to higher problem elaboration.

Figure 6(c) shows eigenvector centrality to be negatively related to problem elaboration. In other words, students who are linked to well-connected others in the network of information seeking performed worse in problem elaboration than students who are not well connected. However, the effect size gets closer to zero when including the interaction between eigenvector and classroom sections, (a)

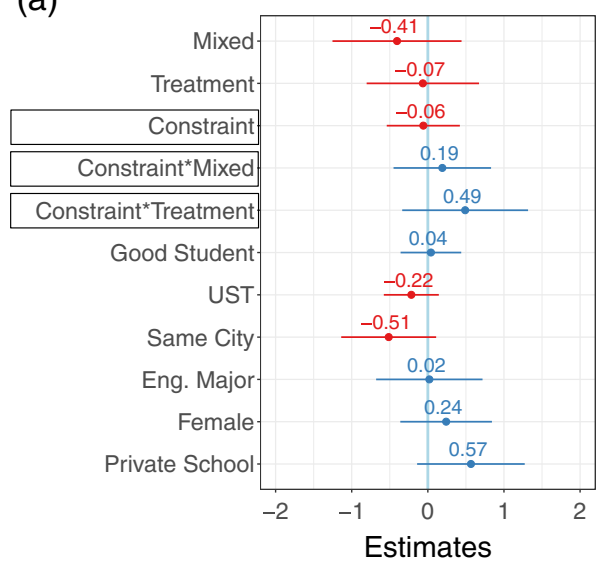

(b)

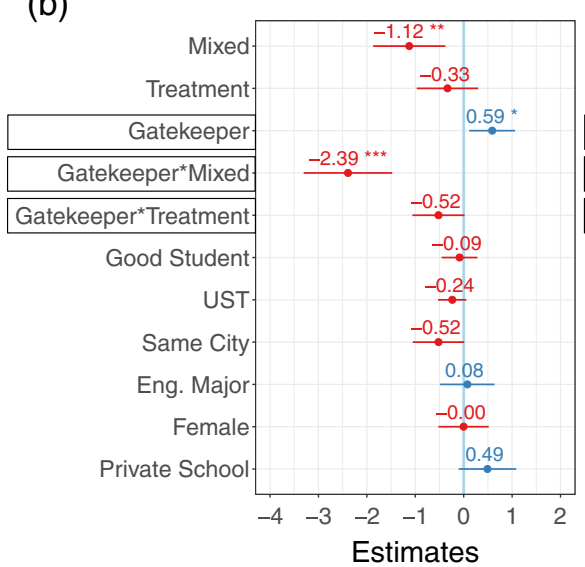

(c)

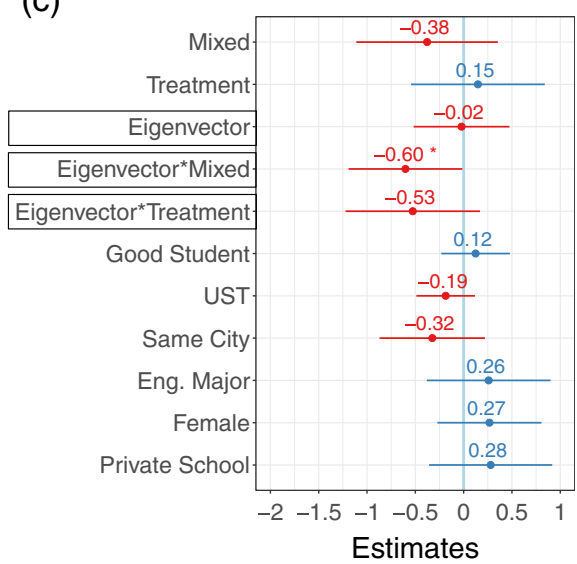

FIG. 6. Graphical depiction of OLS multiple regression models for problem elaboration regressed on network predictors, controlling for confounding variables (see Table 1 in the Supplemental Material [41], results section). Red indicates a negative effect and blue indicates a positive effect. One star $\left(^{*}\right)$ indicates significance at a level of $p$ value $<0.05$ and two stars $(* *)$ indicate significance at a level of $p$ value $<0.01$. 


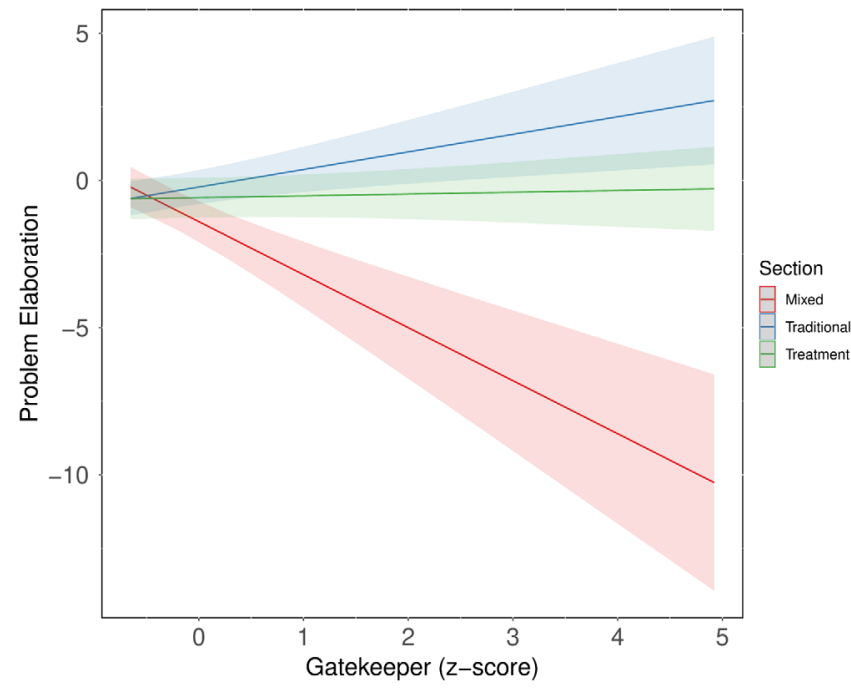

FIG. 7. Linear regression for interaction between gatekeeper and sections for predicting physics grades.

which means that just mixed and treatment sections mainly drive the previously described effect.

\section{The moderation effect of network structures}

Here we explore whether network variables drive the relationship between problem elaboration and physics scores. Because having designed an elaborated physics problem had no direct effect in predicting physics grades (Fig. 4), we considered the possibility that this relationship is moderated by students' structural position within information seeking network. Here we present multiple regression models with moderators in $\log ($ degree) and eigenvector centrality [Figs. 4(d) and 4(e), respectively]. We also tested the moderating effect of perceived good students on the relationship between problem elaboration and physics grades [Fig. 4(f)]. We follow the rationale that different levels of problem elaboration may have enabled differences in conceptual understanding and abilities for solving well-structured problems (i.e., physics grades), at different levels of perceived status (i.e., good students). Both models, Figs. 4(d) and 4(e), had negative coefficients for the interactions between network centralities and problem elaboration in predicting grades. We found the same result for the moderated effect of good student nomination, with a negative coefficient.

Figure 8 depicts the relationship between problem elaboration and physics grades at different levels of log (degree) and good students nomination. First, students who had low degree centrality [red, Fig. 8(a)] benefited from developing problems with high elaboration, as this process afforded them good grades. However, for students with high degree centrality (blue), creating highly elaborated problems had a detrimental effect on grades. Put more simply, scoring high in problem elaboration was associated with good grades only for those who engaged in less social interactions for information seeking [i.e., low log(degree)], which is consistent with our previous results. Finally, Fig. 8(b) depicts the relationship between problem elaboration and physics grades for differing levels of good student nominations. The interaction plot shows that participants who were not perceived as good students in physics (red) benefited from creating well-elaborated problems, which in turn translated into good grades, while good students (blue) might have gotten better grades after creating problems with low levels of elaboration.

\section{DISCUSSION}

Based on the types of problems worked in the mixed section, it is a surprise that the mixed section had lower elaboration than the traditional section [see Fig. 6(b)]. The learning conditions, problems, and instructional guidance on how to solve problems in each section may have influenced students' motivation for creating problems with various levels of elaboration and complexity. For instance, (a)

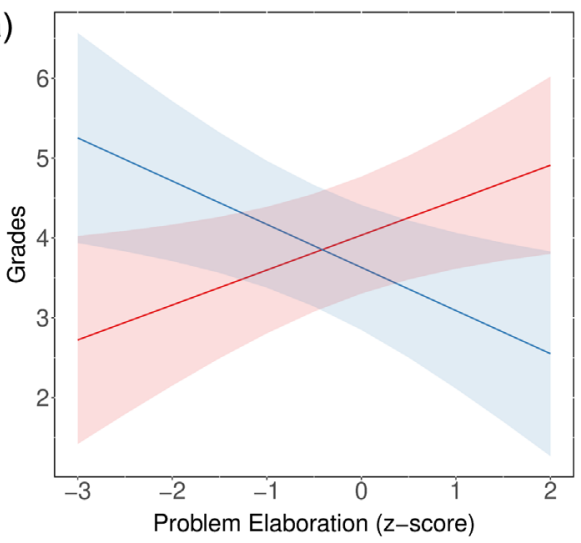

(b)

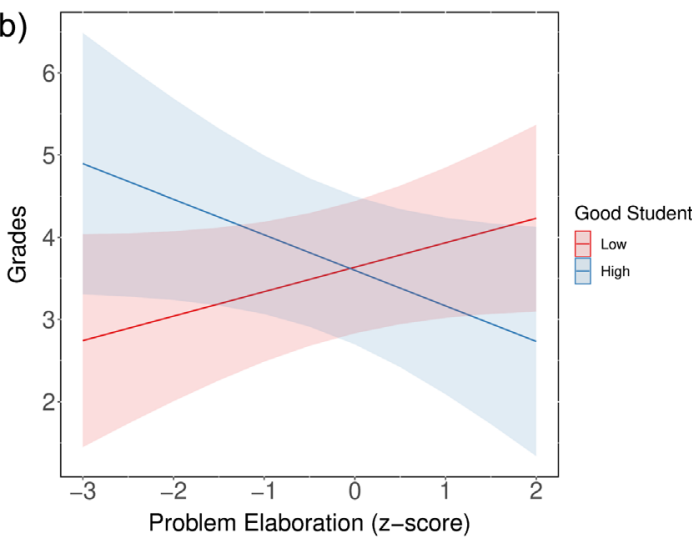

FIG. 8. Interaction between problem elaboration and (a) The logarithm of node degree, and (b) Good student nominations, both from a linear model predicting physics grades. The shaded areas represent a level of confidence interval of 0.9 and low (high) intervals contain the values below (above) the mean of each variable. 
the learning goal of the task (i.e., design a physics problem for secondary students) may have motivated students in the traditional section to utilize characteristics from textbook problems that were in their repository of activities to design problems in an effective way. The mixed section worked on ill-structured problems, but the instructor did not emphasize the importance of assumptions in the face of ill-structured activities. Consequently, we believe that highlighting the role of assumption making when addressing creative tasks had positive effects on students' expectations and motivation for generating problems, as suggested by the high level of problem elaboration found in problems from the treatment section, whose instructor engaged in such a positive narrative for creativity.

Interestingly, being a central actor within the network of information seeking was not related to good grades. This evidence was observed via the relationship between the independent variables such as outdegree, degree, and eigenvector centrality and the dependent variable, and consistent with the evidence found in Candia et al. [7]. The directionality of this relationship contradicts the evidence found in other studies in education and PER [37,38,47]. To understand this contradictory result we argue that the social networks mapped on this and other studies differed in nature, and therefore represent different social processes. For instance, studies in PER had asked students to write down the names of their peers with whom respondents had meaningful interactions inside the classroom $[38,48,49]$. Under such survey question, students are likely to remember interactions with friends [50], or useful interactions related to the learning goals of the session [38]. Differently, the survey question used in this study aimed to determine students' social engagement in the process of seeking out information in the classroom, where students were also likely to report useful as well as friendship-based interactions for information seeking. However, both types of relationships may not necessarily overlap as the nature of the network does not account for the effectiveness of the social tie (i.e., whether the information accessed was useful or not). That is, students may have interacted and reported ties with friends and others not consider friends for information for solving the problem, regardless of the meaningfulness of the interactions. Consequently, and according to the negative relationship found between centrality and physics grades, students were either not capable of requesting appropriate information for solving physics problems due to ineffective communication, or, it may be that engaging in such processes for information seeking is irrelevant in the learning context described here. If the former were true, this would be evidence for the need to engage students in the social processes linked to effective communication and collaboration. Yet, if the learning context were blind to social interactions and information sharing, then this would call for reflecting on the teaching and learning practices involved in university education.
Alternatively, it may be the case that students approximated effective social interactions, yet the actors reached lacked meaningful information to share, or provided misconceptions regarding the content and/or the goals of the task. Consequently, having nodes with reduced knowledge of the content is not an ideal scenario for students to engage in socialization of information for collective growth. This calls for remedial strategies that prepare subjects for proper learning before putting them in positions to collaborate.

It is worth paying attention to the significant interaction between network constraint and sections for predicting physics grades (see Fig. 5). Here, both traditional and treatment were positively related to grades, whereas for the mixed section this relationship is negative. This evidence suggest that the social systems created under traditional and treatment conditions take advantage of highly constrained networks, where subjects presumably engaged in deep analysis and reflection for interrogation of logic [19]. Consequently, within such a cohesive network it is easier to learn complex information as well as to develop good ideas [28]. This process is evidence that the mechanism of creative combinations does not help in solving wellstructured problems, but rather engaging in such efforts brings negative effects. Access to unique portions of the network (i.e., structural holes) is related to inflow of novel ideas, which does not afford better outcomes for solving well-structured problems, likely because the well-bounded nature of the physics information for solving wellstructured problems does not need novelty, but rather conventional knowledge. Further, the negative effect of constraint on the mixed section suggests the opposite, where students benefit from connecting structural holes. Surprisingly, students in the mixed section displayed higher network constraint relative to students from the traditional section (Fig. 7). Consequently, not taking advantage of the mixed section curriculum for scoring higher grades may be due to ineffective communication for collaboration.

Moreover, and even though the models did not yield significant coefficients, constraint had no effect on problem elaboration, whereas it had a negative relationship to physics grades. Differently, while gatekeeper brokerage showed to be a positive predictor for problem elaboration, it was a negative predictor for physics grades. These results add interesting evidence to the contrasting nature of both types of performance, as well as the nature of learning objectives and the measurement instruments designed for such purpose. Generating problems may be similar to benefiting from creative combinations [15] whereas solving well-structured physics problems is not, provided students engaged in effective mechanisms for information seeking in a context that rewards creativity like the treatment section.

The moderated effect of network centrality and good student nomination for predicting physics grades are consistent with the single effect of network structures on physics grades. These results constitute additional evidence 
of the detrimental effect of socialization and seeking out information, presumably through ineffective mechanisms. Surprisingly, students who are not perceived as good students would get better grades by designing problems high in problem elaboration. Alternatively, the complexity of generating physics problems had negative effects for students who enjoyed the social recognition of being proficient in physics. The physics education tradition grounded in wellstructured physics problems $[11,12,22,23]$, and its concordant belief that a good physics performance corresponds to solving well-structured problems has clearly encouraged students to recognize proficient others based on their ability to solve such tasks. However, the set of skills to solve wellstructured problems may not necessarily enable better outcomes in more creatively oriented tasks. This evidence challenges the nature and features of proficiency in this particular context, and pushes us to expand our own perspectives.

\section{LIMITATIONS AND FUTURE RECOMMENDATIONS}

We recognize the limitations of this study associated with the reduced sample size and the lack of alternative variables that would have strengthened the analysis of students' responses in describing their social experience. Further control and observation over instructional strategies would also facilitate a deeper understanding of the nature of the social system generated in each academic section. In addition, short term activities for a single session might discourage interdependency and continued collaboration among students. Consequently, future pedagogical innovations should include higher level structure, with explicit learning goals at individual and group levels. An important dimension for improvement consists of understanding the different ways in which students collaborate and gain access to information from their peers. Such effort might support the interpretation that students engaged in ineffective forms of communication when solving different types of activities, which would lead to recommendations related to the importance of using appropriate strategies for gaining social capital, depending on the nature of the task. Additional evidence on students' strategies for social connection may provide justification for the introduction of pedagogical innovations that lead to creativity and collaboration in university education. Based on these results, educators must be cautious in implementing teaching strategies grounded on principles of collaboration and interdependency. Using such principles would demand intense attention to students' interactions, and appropriate guidance of effective strategies for collaboration and communication of information. In addition, while introducing ill-structured problems in education brings positive learning outcomes and interesting opportunities for creative thinking, novel thinking also occurred when instructors guided the solving process and motivated students to engage in the appropriate cognitive demands these problems entail. In addition, having students develop appropriate content knowledge before attempting to introduce activities that require intense knowledge transfer may induce richer dialogues.

\section{CONCLUSIONS}

Encouraging students to solve open-ended activities such as ill-structured problems in a learning environment that highlights the importance of creativity and socialization of information (i.e., treatment condition) would likely boost students' opportunities for better grades compared to traditional classrooms. Yet, the process of interacting with others for the purpose of accessing new information for solving illstructured problems might be detrimental for obtaining good physics grades, particularly if the latter performance measurement comes from well-structured problems. The nature of the well-structured problems and the features of the learning context tend to reward individualized performance, or collective efforts that emerged from highly cohesive clusters of students. Moreover, well- and ill-structured problems responded positively to different social structures, and therefore, social positions garnered from good grades may have unwanted effects if the tasks are ill structured. Finally, to optimize the learning effects of socialization of information, the learning environment must enable appropriate distribution of knowledge, encourage and value social interactions and the emergence of unconventional ideas, while prioritizing effective communication.

The anonymized data necessary to reproduce this work can be delivered under a reasonable request to the authors. The entire analysis and data processing were done using the standard R libraries [51].

\section{ACKNOWLEDGMENTS}

We acknowledge and appreciate the work of Carlos Rios Morales in facilitating the research setting for conducting this study. We are also thankful of Daniel Katz for his revisions and insights on writing this research article. This material is based upon work supported by the AAPT E. Leonard Jossem International Education Fund. Any opinions, findings, and conclusions or recommendations expressed in this material are those of the author(s) and do not necessarily reflect the views of the American Association of Physics Teachers. 
[1] D. W. Johnson, R. T. Johnson, and E. J. Holubec, Circles of Learning: Cooperation in the Classroom (Interaction Book Company, Edina, MN, 1986).

[2] D. W. Johnson and R. T. Johnson, Cooperation and Competition: Theory and Research (Interaction Book Company, Edina, MN, 1989.

[3] V. Kassarnig, E. Mones, A. Bjerre-Nielsen, P. Sapiezynski, D. D. Lassen, and S. Lehmann, Academic performance and behavioral patterns, EPJ Data Sci. 7, 10 (2018).

[4] D. Blansky, C. Kavanaugh, C. Boothroyd, B. Benson, J. Gallagher, J. Endress, and H. Sayama, Spread of academic success in a high school social network, PLoS One 8, e55944 (2013).

[5] B. Sacerdote, Peer effects in education: How might they work, how big are they and how much do we know thus far?, in Handbook of the Economics of Education, edited by E. Hanushek, S. Machin, and L. Woessmann (North Holland, Amsterdam, 2011), pp. 249-277.

[6] S. Biancani and D. A. McFarland, Social network research in higher education, in Higher Education: Handbook of Theories and Research, edited by M. B. Paulsen (Springer, London, 2013), Chap. 4, pp. 151-215.

[7] C. Candia, V. Landaeta-Torres, C. A. Hidalgo, and C. Rodriguez-Sickert, Strategic reciprocity improves academic performance in public elementary school children. arXiv:1909.11713.

[8] J. W. Pellegrino and M. L. Hilton, Education for Life and Work: Developing Transferable Knowledge and Skills for the 21st Century (National Academies Press, New York, 2003).

[9] D. H. Jonassen, Toward a design theory of problem solving, Educ. Technol. Res. Dev. 48, 63 (2000).

[10] P. Heller and M. Hollabaugh, Teaching problem solving through cooperative grouping. part 2: Designing problems and structuring groups, Am. J. Phys. 60, 637 (1992).

[11] M. T. H. Chi, P. J. Feltovich, and R. Glaser, Categorization and representation of physics problems by experts and novices, Cogn. Sci. 5, 121 (1981).

[12] J. Larkin, J. McDermott, D. P. Simon, and H. A. Simon, Expert and novice performance in solving physics problems, Science 208, 1335 (1980).

[13] D. Fortus, The importance of learning to make assumptions, Sci. Educ. 93, 86 (2008).

[14] N. Shin, D. H. Jonassen, and S. McGee, Predictors of wellstructured and ill-structured problems solving in astronomy simulation, J. Res. Sci. Teach. 40, 6 (2003).

[15] R.S. Burt, Structural holes and good ideas, Am. J. Sociology 110, 349 (2004).

[16] R. S. Burt, The social capital of structural holes, in The New Economic Sociology, edited by M. F Guillen, R Collin, P. England, and M. Meyer (Russell Sage, New York, 2005), pp. 148-192.

[17] R.S. Burt and J. Merluzzi, Network oscillation, Acad. Manage. Disc. 2, 368 (2016).

[18] A. B. Hardagon, Brokering knowledge: linking learning and innovation, Res. Org. Behav. 24, 41 (2002).

[19] L. Rhee and P. Leonardi, Which pathways to good ideas? an intention-based view of innovation in social networks, Strateg. Manag. J. 39, 1188 (2018).
[20] R. J. Dufresne, W. J. Gerace, P. T. Hardiman, and J. P. Mestre, Constraining novices to perform expert-like problem analyses: Effects on schema acquisition, J. Learn. Sci. 2, 307 (1992).

[21] P. B. Kohl and N. D. Finkelstein, Patterns of multiple representations use by experts and novices during physics problem solving, Phys. Rev. Phys. Educ. Res. 4, 010111 (2008).

[22] E. Kim and S. Pak, Students do not overcome conceptual difficulties after solving 1000 traditional problems, Am. J. Phys. 70, 759 (2002).

[23] T. Byun and G. Lee, Why students still can't solve physics problems after solving over 2000 problems, Am. J. Phys. 82, 906 (2014).

[24] I. D. Steiner, Models for inferring relationships between group size and potential productivity, Behav. Sci. 11, 273 (1966).

[25] W. Rietman, Heuristic Decision Procedures, Open Constraints, and the Structure of Ill-Defined Problems (Wiley, New York, 1964).

[26] C. K. W. De Dreu, B. A. Nijstad, M. N. Bechtold, and M. Bass, Group creativity and innovation: A motivated information processing perspective, Psychol. Aesthetics Creativity Arts 5, 81 (2011).

[27] P. Heller, R. Keith, and S. Anderson, Teaching problem solving through cooperative grouping. Part 1: Groups versus individual problem solving, Am. J. Phys. 60, 627 (1992).

[28] L. Fleming, S. Mingo, and D. Cheng, Collaborative brokerage, generative creativity and creative success, Admin. Sci. Quart. 52, 443 (2007).

[29] W. Tsai, Knowledge transfer in intraorganizational networks: Effects of network position and absorptive capacity on business unit innovation and performance, Acad. Management J. 44, 996 (2001).

[30] A. Pentland, Social Physics: How Social Networks Can Make Us Smarter (Penguin Books, New York, 2015).

[31] S. Dawson, J. P. L. Tan, and E. McWilliam, Measuring creative potential: Using social network analysis to monitor a learners' creative capacity, Australas. J. Educ. Tech. 27, 924 (2011).

[32] D. Gašević, A. Zouap, and R. Janzen, Choose your classmates, your GPA is at stake! The association of cross-class social ties and academic performance, Am. Behav. Sci. 57, 1460 (2013).

[33] R. A. Smith and B. L. Peterson, "psst... what do you think"? The relationship between advice prestige, type of advice, and academic performance, Commun. Educ. 56, 278 (2007).

[34] S. P. Borgatti and R. Cross, A relational view of information seeking and learning in social networks, Manage. Sci. 49, 432 (2003).

[35] R. Reagans and B. McEvily, Network structure and knowledge transfer: The effect of cohesion and range, Admin. Quart. Sci. 48, 240 (2003).

[36] A. Calvó-Armengol, E. Patacchini, and Y. Zenou, Peer effects and social networks in education, Rev. Econ. Studies 76, 1239 (2009).

[37] G. Putnik, E. Costa, C. Alves, H. Castro, L. Varela, and V. Shah, Analyzing the correlation between social network analysis measures and performance of students in social 
network-based engineering education, Int. J. Technol. Design Educ. 26, 413 (2016).

[38] J. Bruun and E. Brewer, Talking and learning physics: Predicting future grades from network measures and Force Concept Inventory pretests scores, Phys. Rev. Phys. Educ. Res. 9, 021109 (2013).

[39] J. Pulgar, C. Rios, and C. Candia, Physics problems and instructional strategies for developing social networks in university classrooms, arXiv:1904.02840.

[40] E. Brewe, L. H. Kramer, and G. E. O'Brien, Changing participation through formation of student learning communities, AIP Conf. Proc. 1289, 85 (2010).

[41] See Supplemental Material at http://link.aps.org/ supplemental/10.1103/PhysRevPhysEducRes.16.010137 for experimental protocol, survey items and regression tables.

[42] S. P. Borgatti, M. G. Everett, and J. C. Johnson, Analyzing Social Networks (SAGE, Washington, DC, 2013).

[43] J. Pulgar, A. Spina, C. Ríos, and D. Harlow, Contextual details, cognitive demand and kinematic concepts: Exploring concepts and characteristics of student-generated problems in a university physics course, in Proceedings of the 2019 Physics Education Research Conference, Provo, UT (AIP, New York, 2019).
[44] R. Teodorescu, C. Bennhold, G. Feldman, and L. Medsker, New approach to analyzing physics problems: Ataxonomy of introductory physics problems, Phys. Rev. Phys. Educ. Res. 9, 010103 (2013).

[45] R. S. Burt, Structural Holes (Harvard University Press, Cambridge, MA, 1992).

[46] C. Candia, S. Encarnação, and F. L. Pinheiro, The higher education space: connecting degree programs from individuals' choices, EPJ Data Sci. 8, 39 (2019).

[47] D.Z. Grunspan, B. L. Wiggins, and S. M. Goodreau, Understanding classrooms through social network analysis: A primer for social network analysis in educational research, CBE Life Sci. Educ. 13, 167 (2014).

[48] E. A. Williams, J. P. Zwolak, R. Dou, and E. Brewe, Engagement, integration, involvement: Supporting academic performance and developing a classroom social network., arXiv:1706.04121.

[49] J. P. Zwolak, R. Dou, E. A. Williams, and E. Brewe, Students' network integration as a predictor of persistence in introductory physics courses, Phys. Rev. Phys. Educ. Res. 13, 010113 (2017).

[50] N. Eagle, A. Pentland, and D. Lazer, Inferring friendship network structure using mobile phone data, Proc. Natl. Acad. Sci. U.S.A. 106, 15274 (2009).

[51] https://www.r-project.org/. 